\title{
Blood Cadmium Level Is Associated with Short Progression-Free Survival in Nasopharyngeal Carcinoma
}

\author{
Taifeng Du(D, Wenlong Huang, Shukai Zheng, Mian Bao, Yuanni Huang, Anna Li, Meirong He \\ and Kusheng $\mathrm{Wu} * \mathbb{D}$ \\ Department of Preventive Medicine, Shantou University Medical College, Shantou 515041, China \\ * Correspondence: kswu@stu.edu.cn; Tel.: +86-754-88900445
}

Received: 15 July 2019; Accepted: 14 August 2019; Published: 16 August 2019

\begin{abstract}
The prognosis of nasopharyngeal carcinoma (NPC) is poor with disease progression. Cadmium exposure is a risk factor for NPC. We aimed to investigate the effect of cadmium exposure, by measuring cadmium level, and clinicopathologic factors on NPC disease progression and prognosis. A total of 134 NPC cases were analyzed and venous blood samples were collected. Blood cadmium level was analyzed by graphite furnace atomic absorption spectrophotometry. Clinical data were collected at baseline for patients and tumor characteristics from medical records. Progression-free survival (PFS) was analyzed during follow-up. The effect of cadmium exposure and clinical factors on PFS was analyzed by the Kaplan-Meier method and Cox regression models. Blood cadmium level was associated with history of disease and smoking history and pack-years. On Kaplan-Meier analysis, a high blood cadmium level, male sex, smoking history and increasing pack-years, as well as advanced clinical stage were all associated with short PFS. On multivariate analysis, blood cadmium level was an independent risk factor and predictor of NPC prognosis and disease progression. Cadmium exposure and related clinical factors can affect the prognosis of NPC, which merits further study to clarify.
\end{abstract}

Keywords: Nasopharyngeal carcinoma; progression-free survival time; blood cadmium levels; clinical characteristics; survival analysis

\section{Introduction}

Nasopharyngeal carcinoma (NPC) occurs in epithelial cells of the nasopharynx and belongs to head and neck cancers. Although NPC has similar tissue cells and blood systems as epithelial neoplasms, the two cancers differ in the head and neck region. The incidence of NPC is approximately 87,000 patients/year worldwide, and approximately 50,000 die from NPC annually [1]. As compared with other tumors, NPC is not common and features a geographical distribution trend. The first study of 14 patients combined NPC patients and was published in 1901 [2]. Then, a comprehensive study of 114 patients with a combination of clinical features was published in 1941 [3]. In the United States and Europe, the incidence of NPC is 0.5 to 2/100,000 people [4]. A higher incidence, approximately 80/100,000 people, has been reported in South Asia, parts of the Mediterranean and North Africa [5]. NPC is endemic in Guangdong province, southern China, with an incidence of up to 20 to 30/100,000 people [6].

The etiology of NPC is multifactorial and may be interactive. Possible risk factors include eating salted fish, smoking tobacco, drinking alcohol, exposure to fumes, etc. [7-9]. However, these daily behavioral factors and lifestyles are not sufficient to explain the high incidence of NPC, so there are other unknown risk factors. Occupational exposure to asbestos and cement dust, for example, may increase the risk of NPC [10,11]. Metals are involved in the metabolism of the body for maintaining 
normal life activities. However, an imbalance in element contents can damage the human body. Long-term occupational exposure to heavy metals and smoking can increase heavy metal levels in the blood [12,13]. Tobacco contains many harmful substances, including heavy metals [14,15], which are poisonous and carcinogenic.

Many previous studies found that environmental exposure and occupational exposure to metals are associated with a variety of human cancers. Specifically, decreased selenium (Se) and zinc (Zn) levels may be related to cervical intraepithelial neoplasia and invasive cancer [16]; cadmium (Cd) levels are positively correlated with risk of lung cancer in males [17] and mouth cancer in females [18]; risk of oral cancer is increased with nickel and chromium levels [19]; inorganic arsenic in drinking water is associated with lung and bladder cancers in males [20]; and trace elements have a significant role in the development of carcinoma of larynx [21].

$\mathrm{Cd}$, as a toxic metal, has not been specifically studied and may be involved in the development of NPC [22]. Cd mainly originates from industrial processes such as mining and metal smelting and textile production [23]. People are exposed to Cd mainly through natural contact (such as food, water and dust-containing metal ions) and occupational contact [24]. For non-occupationally exposed people, the primary source of $\mathrm{Cd}$ is diet and smoking tobacco [25]. Tobacco is also an important source of chronic $\mathrm{Cd}$ exposure. The human body absorbs approximately $3 \mu \mathrm{g} \mathrm{Cd}$ by lung absorption when smoking a pack of cigarettes per day [26]. Long-term exposure to Cd causes harm to human health when the accumulation of elements in human body increases beyond the degradation ability of the body and it is difficult to excrete them. Previous studies have found that higher $\mathrm{Cd}$ levels are associated with the occurrence of other types of cancer, such as endometrial cancer [27], bladder cancer [28], pancreatic cancer [29], and postmenopausal breast cancer [30]. Low to moderate Cd exposure was prospectively associated with total cancer mortality of the lung and pancreas [31]. Therefore, the harm of $\mathrm{Cd}$ to the human body and the occurrence of tumors is a concern.

Although research on the pathogenesis of $\mathrm{Cd}$ in the body is relatively scarce, because of its long-term accumulation, Cd level is likely highly correlated with the prognosis of a tumor such as NPC and its progression. Many factors affect disease progression of NPC, and there may be an interaction between various factors. Disease progression refers to a $20 \%$ increase in the sum of the longest diameters of all target lesions and is not considered with increase in size of just one lesion. In addition to the expansion of local lesions, the other type of disease progression is transfer to another part. These two situations can be found mainly on imaging. Actually, clinical deterioration belongs to disease progression and is characterized by tumor spread and metastasis, disease recurrence, nosebleed, and lymph node transfer in the head and neck. The potential factors affecting the survival and prognosis of NPC can generally be divided into internal and external factors. The status of tumor cells themselves such as the nature of tumor cells and surrounding stroma may also be an important factor for clinical progression [32]. Moreover, previous studies showed that clinical stage, sex, smoking history and T classification are significantly associated with the survival and prognosis of NPC [33-35]. These factors may be related to distant metastasis and progression of NPC.

Previous studies have found that $\mathrm{Cd}$ level is possibly associated with the development of NPC $[22,36,37]$. In the present study, to explore the prognostic factors of NPC and further predict NPC progression, clinical information and baseline data were collected and the blood Cd level of patients was measured to explore the possible associations between these factors and NPC progression and prognosis.

\section{Materials and Methods}

\subsection{Subjects}

In December 2014, from our medical records, we selected patients with a diagnosis of NPC from 1 August 2002 to 18 February 2014 at the Cancer Hospital in Shantou city. We included patients who were (1) receiving similar treatment (cisplatin and paclitaxel chemotherapy), (2) had no occupational 
exposure to Cd, and (3) had histopathologically confirmed NPC. The incidence of NPC is as high as 30/100,000 people in southern China, especially in Guangdong province [6], so most of the patients included were from Chaoshan area, which is located in the eastern coastal area of Guangdong province, and the rest were from neighboring areas. After being informed of the specific purpose of the study, all patients were asked to complete an informed consent form. Blood samples were taken within 3 days after the histopathologic diagnosis of NPC. When informed consent was obtained, $2 \mathrm{~mL}$ venous blood was obtained from patients by a trained nurse, then put into K-EDTA (ethylene diamine tetraacetic acid) metal-free vials, and stored at $-80^{\circ} \mathrm{C}$. All plastic tubes and containers used for the determination of blood Cd levels were washed, soaked in nitric acid, washed for deionization and dried before use to avoid contamination. The study was approved by the human ethics committee of Shantou University Medical College. The medical records and pathology reports of patients are available if needed.

\subsection{Collection of General and Clinical Data}

Relevant general, pathological and clinical data were obtained from medical records following the procedures and guidelines of the Cancer Hospital in Shantou city. General information included age, sex, smoking, alcohol drinking, residence, occupation, etc. Relevant general information was obtained from medical records and by face-to-face and telephone interview. For the cumulative smoking dose, we divided patients into four groups by pack-years of smoking: no smoking, $<30,30$ to 40 , and $\geq 40$ pack-years. Clinical data included age of diagnosis, family history of cancer, and history of disease (including hypertension, diabetes, asthma, kidney stones, etc.). Pathological information mainly included tumor-node-metastasis (T, N, M) classification; Epstein-Barr virus antibody levels (early antigen (EA), viral capsid antigen antibody (VCA)); clinical stage; and pathological type.

$\mathrm{T}, \mathrm{N}$ and $\mathrm{M}$ classification were classified according to the TNM system of the International Union for Cancer Control and the American Joint Committee on Cancer. T classification is divided into four groups by site of tumor spread: T1 (limited to the nasopharynx), T2 (spread to the nasal tissue), T3 (spread to bony structures), and T4 (spread to the brain or brain nerve). $\mathrm{N}$ classification is divided into four groups by the diameter of lymph nodes metastasized: N0 (no lymph node metastasis), $\mathrm{N} 1$ (largest diameter $\leq 3 \mathrm{~cm}), \mathrm{N} 2(3-6 \mathrm{~cm})$, and N3 $(>6 \mathrm{~cm})$. M classification is divided into two groups by whether the tumor has distant metastasis: M0 (no distant metastasis) and M1 (distant metastasis).

\subsection{Collection of Survival Time Data}

All patients were interviewed after diagnosis and treatment at the cancer hospital in Shantou city. We collected patient information from medical records. To conduct a follow-up, we set the baseline time of NPC diagnosis from 1 August 2002 to 18 February 2014 and the endpoint as 31 December 2014. Disease progression was defined as the disease recurrence, metastasis or death. Survival time was defined as the cancer-free period from the start of diagnosis and treatment at the hospital to the onset of disease progression. If no disease progression was observed at the endpoint, these data were censored. During the follow-up period, the survival time was defined as full data if the patient died or disease progression occurred. Survival time was a significant indicator for evaluating disease progression.

\subsection{Analysis of Blood Cd Level}

Blood samples were used to analyze the risk of $\mathrm{Cd}$ exposure in patients. Blood $\mathrm{Cd}$ levels were measured by a standardized method also used in other publications [38]. A volume of $200 \mu \mathrm{L}$ blood sample was placed in a tube containing $800 \mu \mathrm{L} 5 \%$ nitric acid. After vortexing, the sample was digested for $10 \mathrm{~min}$, then centrifuged for $15 \mathrm{~min}$ at $3000 \mathrm{rpm}$ to separate the supernatant. The whole experiment was conducted in the laboratory of environmental medicine, Shantou University Medical College. For the quantitative analysis of $\mathrm{Cd}$ in blood, the peak area was determined by graphite furnace atomic absorption spectrophotometry (Jena ZEEnit 650, Analytik Jena, Jena, Germany), and the injection volume was set to $20 \mu \mathrm{L}$. The main parameters of the experiment were as follows: a lamp current of $4.0 \mathrm{~mA}$; a width of $1.2 \mathrm{~nm}$; a wavelength of $228.8 \mathrm{~nm}$; drying temperature at 90,105 , and $120{ }^{\circ} \mathrm{C}$; 
pyrolyzing and atomization at 300 and $1300{ }^{\circ} \mathrm{C}$, respectively. The limits of detection (LODs) were calculated to be $0.01 \mu \mathrm{g} / \mathrm{L}$. The results of measurement accuracy showed recoveries within 100 and $103 \%$ by analyzing the spiked samples $(n=8)$. That the precision was better than $5.5 \%$ indicated good repeatability for cadmium detection. In order to ensure the quality control and assurance of the experiment, we used standard solutions, analytical duplicates, and reagent blanks to detect drift, precision, and signs of contamination in turn. The linear correlation coefficient of the cadmium standard calibration curve was good, reaching 0.9964. All glassware and plastic in this study were soaked in $20 \%(\mathrm{v} / \mathrm{v}) \mathrm{HNO}_{3}$ for over $24 \mathrm{~h}$ and rinsed three times with deionized water.

\subsection{Statistical Analysis}

Data were analyzed by using SPSS 23.0 (SPSS Inc., Chicago, IL, USA). Data with normal distribution are represented by the mean \pm SD and were analyzed by an independent-sample $t$ test. Data with non-normal distribution are represented by median (interquartile range (IQR)) and were analyzed by the Mann-Whitney U test and Kruskal-Wallis $\mathrm{H}$ test. Categorical variables were analyzed by the chi-square test. The data for blood $\mathrm{Cd}$ level were divided into two groups by median concentration $(3.84 \mathrm{mg} / \mathrm{L})$, named high level and low level, respectively. Survival time was defined as the time between the diagnosis of NPC and the discovery of progressive NPC or death. Kaplan-Meier log-rank tests were used to analyze survival time by various factors, and median survival time was also estimated by survival analysis. Progression-free survival (PFS) was analyzed by the variables age at diagnosis; family history of cancer; history of disease; T, $\mathrm{M}$ and $\mathrm{N}$ classification; clinical stage; $\mathrm{EA}$ and VCA positivity; pathological type; and blood Cd level by Cox proportional-hazard regression models, estimating hazard ratios (HRs) and 95\% confidence intervals (CIs). Spearman rank correlation analysis was also used to evaluate the relationships between investigated factors and blood Cd level with NPC. All tests were two-sided and $p<0.05$ was considered statistically significant.

\section{Results}

\subsection{Association between Clinical Characteristics and NPC Progression}

We included 134 patients with NPC (76.9\% females; mean age $55.57 \pm 11.93$ years). The median progression-free survival (PFS) for all patients was 2 months and 49 patients showed NPC progression: eight $(16.3 \%)$ had a family history of cancers, $35(71.4 \%)$ had a smoking history, and eight $(16.3 \%)$ had a drinking history (Table 1). The proportion of patients with disease progression was higher with than without a history of smoking $(71.4 \%$ vs. $28.6 \% ; p=0.018$; Table 1$)$. In addition, the proportion of relatively high median blood $\mathrm{Cd}$ level was higher with than without disease progression (7.62 (IQR 3.37-9.43) vs. $3.09(1.77-4.80) \mu \mathrm{g} / \mathrm{L} ; p<0.001$, Table 1).

Table 1. Clinicopathologic characteristics of the participants with and without nasopharyngeal carcinoma (NPC) progression $(n=134)$.

\begin{tabular}{|c|c|c|c|}
\hline $\begin{array}{l}\text { Clinicopathologic } \\
\text { Characteristics }\end{array}$ & $\begin{array}{l}\text { NPC Progression } \\
n=49\end{array}$ & $\begin{array}{l}\text { No NPC Progression } \\
\mathrm{n}=85\end{array}$ & $p$-Value \\
\hline Age (years), mean \pm SD & $54.00 \pm 11.61$ & $56.47 \pm 12.08$ & $0.250^{\mathrm{a}}$ \\
\hline Cd level $(\mu \mathrm{g} / \mathrm{L})$, median, IQR & $7.62(3.37-9.43)$ & $3.09(1.77-4.80)$ & $<0.001 *$ \\
\hline EA+ & $4.53(0.73-10.08)$ & $5.99(2.51-10.36)$ & $0.270^{\mathrm{a}}$ \\
\hline $\mathrm{VCA}+$ & $0.37(0.03-2.05)$ & $1.15(0.21-3.09)$ & $0.092^{\mathrm{a}}$ \\
\hline \multicolumn{4}{|l|}{ Sex } \\
\hline Female & $8(16.3)$ & $23(27.1)$ & $0.156^{\mathrm{b}}$ \\
\hline Male & $41(83.7)$ & $62(72.9)$ & \\
\hline \multicolumn{4}{|l|}{ Family history of cancer } \\
\hline Yes & $8(16.3)$ & 15 (17.6) & $0.845^{b}$ \\
\hline No & $41(83.7)$ & $70(82.4)$ & \\
\hline
\end{tabular}


Table 1. Cont.

\begin{tabular}{|c|c|c|c|}
\hline $\begin{array}{l}\text { Clinicopathologic } \\
\text { Characteristics }\end{array}$ & $\begin{array}{l}\text { NPC Progression } \\
n=49\end{array}$ & $\begin{array}{l}\text { No NPC Progression } \\
n=85\end{array}$ & $p$-Value \\
\hline \multicolumn{4}{|l|}{ History of disease } \\
\hline Yes & $12(24.5)$ & $33(38.8)$ & $0.091^{b}$ \\
\hline No & $37(75.5)$ & $52(61.2)$ & \\
\hline \multicolumn{4}{|l|}{ Smoking pack-years } \\
\hline No smoking & $14(28.6)$ & $42(49.4)$ & $0.118^{b}$ \\
\hline$<30$ & $12(24.5)$ & $17(20.0)$ & \\
\hline $30-40$ & $8(16.3)$ & $10(11.8)$ & \\
\hline$\geq 40$ & $15(30.6)$ & $16(18.8)$ & \\
\hline \multicolumn{4}{|l|}{ Smoking history } \\
\hline Yes & $35(71.4)$ & $43(50.6)$ & $0.018^{b}$ \\
\hline No & $14(28.6)$ & $42(49.4)$ & \\
\hline \multicolumn{4}{|l|}{ Alcohol drinking } \\
\hline Yes & $8(16.3)$ & $10(11.8)$ & $0.456^{\mathrm{b}}$ \\
\hline No & $41(83.7)$ & $75(88.2)$ & \\
\hline \multicolumn{4}{|l|}{ T classification } \\
\hline $\mathrm{T} 1$ & $5(10.4)$ & $1(1.2)$ & $0.080^{\mathrm{b}}$ \\
\hline $\mathrm{T} 2$ & $14(29.2)$ & $32(37.6)$ & \\
\hline T3 & $13(27.1)$ & $20(23.5)$ & \\
\hline $\mathrm{T} 4$ & $16(33.3)$ & $32(37.6)$ & \\
\hline \multicolumn{4}{|l|}{ Clinical stages } \\
\hline Early & $1(2.0)$ & $8(9.4)$ & $0.101^{b}$ \\
\hline Advanced & $48(98.0)$ & $77(90.6)$ & \\
\hline \multicolumn{4}{|l|}{$\mathrm{N}$ classification } \\
\hline No & $2(4.2)$ & $3(3.5)$ & $0.573^{b}$ \\
\hline N1 & $5(10.4)$ & $14(16.5)$ & \\
\hline N2 & $34(70.8)$ & $61(71.8)$ & \\
\hline N3 & $7(14.6)$ & $7(8.2)$ & \\
\hline \multicolumn{4}{|l|}{ M classification } \\
\hline M0 & $44(91.7)$ & 77 (90.6) & $0.835^{b}$ \\
\hline M1 & $4(8.3)$ & $8(9.4)$ & \\
\hline \multicolumn{4}{|l|}{ Pathological types } \\
\hline Undifferentiated & $36(73.5)$ & $50(58.8)$ & $0.234^{b}$ \\
\hline Differentiated & $12(24.5)$ & $32(37.6)$ & \\
\hline Squamous & $1(2.0)$ & $3(3.5)$ & \\
\hline
\end{tabular}

Smoking pack-years, [cigarettes per day/20] $\times$ years smoked; T classification, size of the primary tumor; N classification, lesion of regional lymph nodes; Cd, cadmium; IQR, interquartile range; EA, early antigen; VCA, viral capsid antigen antibody. ${ }^{*}$ Mann-Whitney U test due to non-normal distribution; ${ }^{\mathrm{a}}$ Independent sample $t$ tests; ${ }^{\mathrm{b}}$ chi-square test.

\subsection{Association between Clinical Characteristics of NPC Patients and Blood Cd Level}

Blood Cd level was related to the history of disease $(p=0.017)$, smoking history $(p=0.014)$, clinical stage $(p=0.016)$, and smoking pack-years $(p=0.003)$ (Table 2). For further analysis, we divided the blood Cd level into high level $(\geq 3.84 \mu \mathrm{g} / \mathrm{L})$ and low level $(<3.84 \mu \mathrm{g} / \mathrm{L})$ according to the median concentration. Probability of high $\mathrm{Cd}$ level was associated with smoking $\geq 40$ pack-years versus not smoking ( $\mathrm{OR}=4.83,95 \%$ CI 1.68-13.91, $p=0.004$ ) (Table 3). Probability of low Cd level was associated with history of disease versus no history (OR $=0.39,95 \%$ CI $0.17-0.89, p=0.025)$. Additionally, probability of high Cd level was associated with advanced disease stage but not significantly. 
Table 2. Association between clinicopathological features and blood Cd levels with NPC $(\mathrm{n}=134)$.

\begin{tabular}{|c|c|c|c|c|}
\hline \multirow{2}{*}{$\begin{array}{l}\text { Clinicopathologic } \\
\text { Characteristics }\end{array}$} & \multirow{2}{*}{ No. of Patients } & \multicolumn{2}{|c|}{ Blood Cd Level } & \multirow{2}{*}{$p$-Value } \\
\hline & & $<3.84 \mu \mathrm{g} / \mathrm{L}(\mathrm{n}=67)$ & $\geq 3.84 \mu \mathrm{g} / \mathrm{L}(\mathrm{n}=67)$ & \\
\hline Age (years), mean \pm SD & 134 & $55.40 \pm 11.66$ & $55.73 \pm 12.28$ & $0.631^{\mathrm{a}}$ \\
\hline $\mathrm{EA}+$ & 106 & $5.13(1.73-9.97)$ & $7.10(2.49-10.71)$ & $0.185^{\mathrm{a}}$ \\
\hline $\mathrm{VCA}+$ & 107 & $0.81(0.12-2.40)$ & $0.89(0.21-3.35)$ & $0.432^{\mathrm{a}}$ \\
\hline \multicolumn{5}{|l|}{ Sex } \\
\hline Female & 103 & $49(73.1)$ & $54(80.6)$ & \multirow[t]{2}{*}{$0.306^{\mathrm{b}}$} \\
\hline Male & 31 & $18(26.9)$ & $13(19.4)$ & \\
\hline \multicolumn{5}{|l|}{ Family history of cancer } \\
\hline Yes & 23 & 8 (11.9) & $15(22.4)$ & \multirow[t]{2}{*}{$0.109^{b}$} \\
\hline No & 111 & $59(88.1)$ & $52(77.6)$ & \\
\hline \multicolumn{5}{|l|}{ Disease history } \\
\hline Yes & 45 & $29(43.3)$ & $16(23.9)$ & \multirow[t]{2}{*}{$0.017^{b}$} \\
\hline No & 89 & $38(56.7)$ & $51(76.1)$ & \\
\hline \multicolumn{5}{|l|}{ Smoking pack-years } \\
\hline No smoking & 56 & $35(52.2)$ & $21(31.3)$ & \multirow[t]{4}{*}{$0.003^{b}$} \\
\hline$\geq 30$ & 29 & $17(25.4)$ & $12(17.9)$ & \\
\hline $30-40$ & 18 & $8(11.9)$ & $10(14.9)$ & \\
\hline$\geq 40$ & 31 & $7(10.4)$ & $24(35.8)$ & \\
\hline \multicolumn{5}{|l|}{ Smoking history } \\
\hline Yes & 78 & $32(47.8)$ & $46(68.7)$ & \multirow[t]{2}{*}{$0.014^{\mathrm{b}}$} \\
\hline No & 56 & $35(52.2)$ & $21(31.3)$ & \\
\hline \multicolumn{5}{|l|}{ Alcohol drinking } \\
\hline Yes & 18 & $7(10.4)$ & $11(16.4)$ & \multirow[t]{2}{*}{$0.311^{b}$} \\
\hline No & 116 & $60(89.6)$ & $56(83.6)$ & \\
\hline \multicolumn{5}{|l|}{ Clinical stage } \\
\hline Early & 9 & $8(11.9)$ & $1(1.5)$ & \multirow[t]{2}{*}{$0.016^{\mathrm{b}}$} \\
\hline Advanced & 125 & $59(88.1)$ & $66(98.5)$ & \\
\hline \multicolumn{5}{|l|}{ T classification } \\
\hline $\mathrm{T} 1$ & 6 & $2(3.0)$ & $4(6.1)$ & \multirow[t]{4}{*}{$0.356^{\mathrm{b}}$} \\
\hline $\mathrm{T} 2$ & 46 & $25(37.3)$ & $21(31.8)$ & \\
\hline T3 & 33 & $13(19.4)$ & $20(30.3)$ & \\
\hline $\mathrm{T} 4$ & 48 & $27(40.3)$ & $21(31.8)$ & \\
\hline \multicolumn{5}{|l|}{$\mathrm{N}$ classification } \\
\hline No & 5 & $3(4.5)$ & $2(3.0)$ & \multirow[t]{4}{*}{$0.345^{b}$} \\
\hline N1 & 19 & $13(19.4)$ & $6(9.1)$ & \\
\hline N2 & 95 & $45(67.2)$ & $50(75.8)$ & \\
\hline N3 & 14 & $6(9.0)$ & $8(12.1)$ & \\
\hline \multicolumn{5}{|l|}{ M classification } \\
\hline M0 & 121 & $62(92.5)$ & $59(89.4)$ & \multirow[t]{2}{*}{$0.527^{b}$} \\
\hline M1 & 12 & $5(7.5)$ & $7(10.6)$ & \\
\hline Pathological types & & & & \\
\hline Undifferentiated & 86 & $40(59.7)$ & $46(68.7)$ & $0.410^{b}$ \\
\hline Differentiated & 44 & $24(35.8)$ & $20(29.9)$ & \\
\hline Squamous & 4 & $3(4.5)$ & $1(1.5)$ & \\
\hline
\end{tabular}

Smoking pack-years, [cigarettes per day/20] $\times$ years smoked; $\mathrm{T}$ classification, size of the primary tumor; $\mathrm{N}$ classification, lesion of regional lymph nodes; Cd, cadmium; EA, early antigen; VCA, viral capsid antigen antibody. ${ }^{\mathrm{a}}$ Independent sample $t$ tests; ${ }^{\mathrm{b}}$ chi-square test. 
Table 3. Multivariate analysis of factors associated with blood Cd level for NPC patients $(n=134)$.

\begin{tabular}{|c|c|c|c|c|}
\hline \multirow[b]{2}{*}{ Variables } & \multicolumn{2}{|c|}{ Blood Cd Level } & \multirow[b]{2}{*}{ OR $(95 \% C I)$} & \multirow{2}{*}{$p$-Value } \\
\hline & $\begin{array}{l}<3.84 \mu \mathrm{g} / \mathrm{L} \\
\mathrm{n}=67\end{array}$ & $\begin{array}{l}\geq 3.84 \mu \mathrm{g} / \mathrm{L} \\
\mathrm{n}=67\end{array}$ & & \\
\hline \multicolumn{5}{|c|}{ Family History of Cancer } \\
\hline No & $59(88.1)$ & $52(77.6)$ & Reference & \\
\hline Yes & $8(11.9)$ & $15(22.4)$ & $1.52(0.53-4.40)$ & 0.437 \\
\hline \multicolumn{5}{|c|}{ Smoking pack-years } \\
\hline No smoking & $35(52.2)$ & $21(28.8)$ & Reference & \\
\hline$<30$ & $17(25.4)$ & $12(16.4)$ & $1.14(0.44-2.96)$ & 0.790 \\
\hline $30-40$ & $8(11.9)$ & $16(21.9)$ & $2.01(0.64-6.32)$ & 0.228 \\
\hline$\geq 40$ & $7(10.4)$ & $24(32.9)$ & $4.83(1.68-13.91)$ & 0.004 \\
\hline \multicolumn{5}{|c|}{ History of disease } \\
\hline No & $38(56.7)$ & $51(76.1)$ & Reference & \\
\hline Yes & $29(43.3)$ & $16(23.9)$ & $0.39(0.17-0.89)$ & 0.025 \\
\hline \multicolumn{5}{|l|}{ Clinical stage } \\
\hline Early & $8(11.9)$ & $1(1.5)$ & Reference & \\
\hline Advanced & $59(88.1)$ & $66(98.5)$ & $5.50(0.62-48.54)$ & 0.125 \\
\hline
\end{tabular}

OR, odds ratio; 95\% CI, 95\% confidence interval; Smoking pack-years, [cigarettes per day/20]×years smoked.

Blood Cd level was positively correlated with clinical stage $(\mathrm{r}=0.192, p<0.05)$, smoking pack-years $(\mathrm{r}=0.314, p<0.01)$, and smoking history $(\mathrm{r}=0.224, p<0.01)$ (Table 4$)$. We found a negative correlation between blood $\mathrm{Cd}$ level and history of disease $(\mathrm{r}=-0.202 ; p<0.05)$. 
Table 4. Spearman correlation coefficients between investigated factors and blood Cd level with NPC ( $n=134)$.

\begin{tabular}{|c|c|c|c|c|c|c|c|c|c|c|c|}
\hline Investigated Factors & SPY & Age & Sex & FHC & DH & $\mathrm{AD}$ & CS & PT & $\mathrm{SH}$ & BCL & VCA+ \\
\hline EA+ & -0.025 & 0.204 * & -0.108 & -0.045 & -0.129 & $-0.268^{* *}$ & 0.032 & $0.207^{*}$ & -0.080 & 0.167 & $0.476^{\text {** }}$ \\
\hline VCA+ & -0.082 & 0.149 & 0.179 & -0.039 & -0.014 & -0.066 & -0.095 & -0.105 & -0.100 & 0.065 & \\
\hline $\mathrm{BCL}$ & $0.314^{* *}$ & -0.006 & -0.056 & 0.146 & $-0.202 *$ & -0.088 & 0.192 * & -0.155 & $0.224^{* *}$ & & \\
\hline $\mathrm{SH}$ & $0.899 * *$ & 0.064 & $-0.612^{* *}$ & 0.145 & -0.006 & $0.289^{* *}$ & 0.135 & 0.017 & & & \\
\hline PT & 0.031 & 0.017 & -0.074 & 0.020 & -0.051 & -0.100 & -0.003 & & & & \\
\hline CS & 0.143 & -0.064 & -0.065 & 0.122 & -0.125 & 0.018 & & & & & \\
\hline $\mathrm{AD}$ & $0.269 * *$ & 0.048 & $-0.216^{*}$ & 0.111 & $0.183^{*}$ & & & & & & \\
\hline $\mathrm{DH}$ & -0.023 & 0.056 & -0.053 & 0.137 & & & & & & & \\
\hline FHC & $0.241^{* *}$ & 0.107 & -0.109 & & & & & & & & \\
\hline Sex & $-0.539 * *$ & -0.039 & & & & & & & & & \\
\hline Age & $0.204 *$ & & & & & & & & & & \\
\hline
\end{tabular}
early antigen; VCA, viral capsid antigen antibody. ${ }^{*} p<0.05 ;{ }^{* *} p<0.01$ 


\subsection{Prognostic Factors Associated with PFS and NPC Progression}

Kaplan-Meier analysis showed that smoking history, sex, blood Cd level, smoking pack-years, and clinical stage are all associated with PFS (log-rank test; all $p<0.05$; Figure 1). PFS was shorter for males than females $(p=0.039)$ and with than without a smoking history $(p=0.004)$. With increasing smoking pack-years and clinical stage, PFS decreased $(p=0.031$, and 0.040 , respectively). Moreover, high blood Cd level was strongly associated with short PFS $(p<0.001)$. Nevertheless, short PFS was not associated with age at diagnosis, family history of cancer, alcohol drinking, pathological type, type of Epstein-Barr virus antibody (EA, VCA), or T, N, M classification (Figure 2).

High blood Cd level was a significant prognostic risk factor for NPC progression (HR $=3.76 ; 95 \%$ CI 1.75-8.06; $p=0.001$; Table 5).
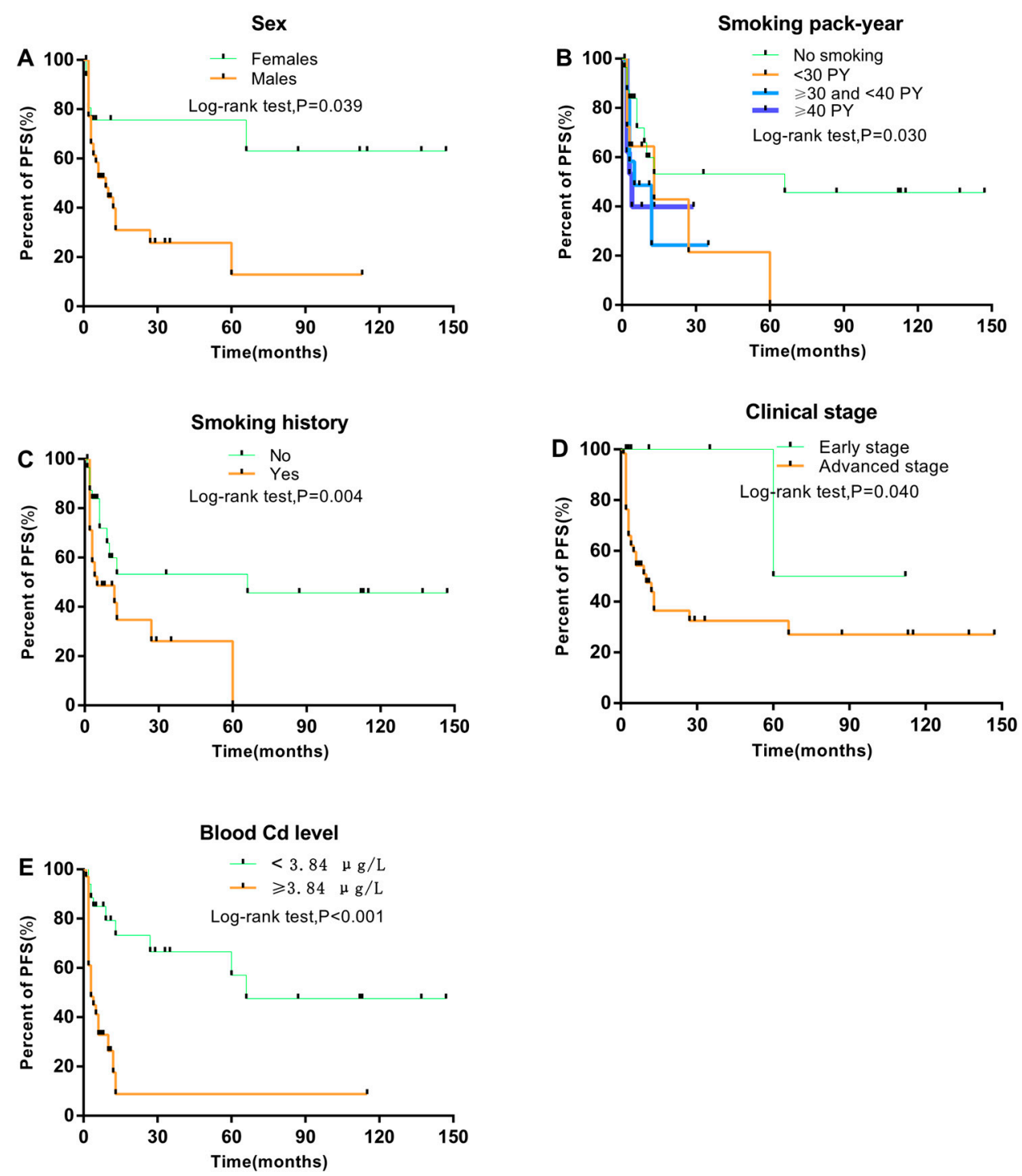

Figure 1. Comparison of PFS among different groups of NPC patients by Kaplan-Meier log-rank test. (A-E) PFS by sex, smoking pack-years, smoking history, clinical stage, and blood Cd level, respectively $(\mathrm{n}=134)$. 

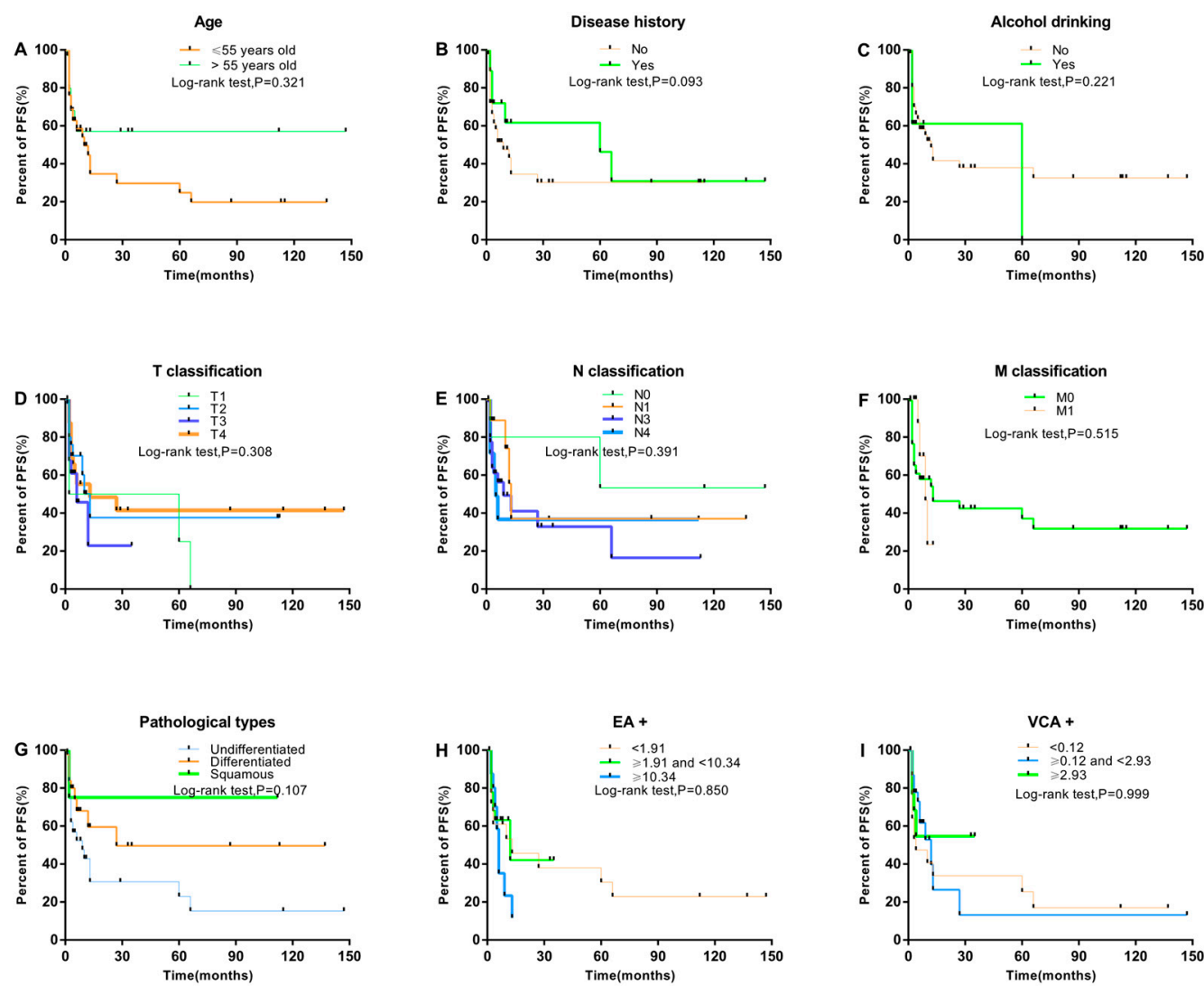

Figure 2. Comparison of PFS among different groups of NPC patients by Kaplan-Meier log-rank test. (A-I) PFS by age, disease history, alcohol drinking, T classification, $\mathrm{N}$ classification, M classification, pathological type, early antigen-positive $(\mathrm{EA}+)$, and VCA, viral capsid antigen antibody-positive $(\mathrm{VCA}+)$, respectively $(\mathrm{n}=134)$.

Table 5. Proportional-hazards analysis of factors associated with progression-free survival (PFS) with NPC $(n=134)$.

\begin{tabular}{|c|c|c|c|}
\hline \multirow{2}{*}{ Variables } & \multicolumn{3}{|c|}{ PFS } \\
\hline & HR & $95 \%$ CI & $p$-Value \\
\hline Sex (male vs. female) & 1.25 & $0.45-3.42$ & 0.671 \\
\hline \multicolumn{4}{|l|}{ Residence } \\
\hline Chaozhou & Reference & & \\
\hline Shantou & 0.70 & $0.28-1.74$ & 0.443 \\
\hline Jieyang & 0.99 & $0.39-2.48$ & 0.976 \\
\hline Other area & 0 & 0 & 0.976 \\
\hline Family history of cancer (yes vs. no) & 1.11 & $0.49-2.53$ & 0.800 \\
\hline History of disease (yes vs. no) & 0.65 & $0.31-1.34$ & 0.240 \\
\hline Alcohol drinking (yes vs. no) & 1.11 & $0.46-2.71$ & 0.817 \\
\hline \multicolumn{4}{|l|}{ Smoking pack-years } \\
\hline No smoking & Reference & & \\
\hline$<30$ & 2.32 & $0.87-6.22$ & 0.093 \\
\hline $30-40$ & 1.47 & $0.52-4.18$ & 0.468 \\
\hline$\geq 40$ & 1.76 & $0.70-4.42$ & 0.230 \\
\hline Clinical stage (advanced vs early) & 3.03 & $0.37-24.65$ & 0.301 \\
\hline Blood Cd level ( $\geq 3.84$ vs. $<3.84 \mu \mathrm{g} / \mathrm{L})$ & 4.11 & $1.92-8.81$ & $<0.001$ \\
\hline
\end{tabular}

HR, hazard ratio; Smoking pack-years, [cigarettes per day/20] $\times$ years smoked. 


\section{Discussion}

The present study assessed the prognosis of NPC in patients by analyzing blood Cd level and clinicopathological data. Blood $\mathrm{Cd}$ level was positively correlated with $\mathrm{N}$ classification, smoking history, and smoking pack-years and negatively with disease history. High blood Cd level, male sex, smoking history and increasing pack-years as well as advanced clinical stage were significantly associated with short PFS. In addition, high blood Cd level was an independent prognostic factor for short PFS. Clinicopathologic factors and blood Cd level may affect the prognosis of NPC, and blood $\mathrm{Cd}$ level could be a novel risk factor for NPC progression.

Patient sex, smoking history, and clinical stages were all prognostic factors of NPC, but no association was found with drinking history, pathological type, $\mathrm{N}$ classification, and radiotherapy pattern in previous studies [32,39]. Sex can affect the curative effect of NPC patients, and men were found to have a higher rate of metastasis and disease progression than women, which is consistent with our results $[40,41]$. Sex was an important prognostic factor, and female NPC patients were previously found to have a higher survival rate than males (80.2 and 71.9\%) [32]. Smoking is an important factor in tumor development and causes normal epithelial cell tumors to develop as mutagen and DNA damage agents [42]. Several studies have evaluated the effect of tobacco use in head and neck cancer, including NPC. Tobacco use decreased head and neck cancer survival during radiotherapy or medication [43]. A follow-up study showed smoking status as an important predictor of survival in NPC patients [44]. NPC patients who gave up smoking had a higher survival rate than those who currently smoked [45]. Of course, smoking pack-years may also be associated with the survival and prognosis of NPC because smoking time and quantity accumulate.

The 5-year survival rate is higher in the early clinical stages (stages I and II) of NPC patients [46,47]. However, over half of the patients are diagnosed at advanced stages, which greatly reduce the survival rate [48]. The tumor has no obvious symptoms at the early stage; symptoms are mostly observed in the advanced stage. Cheng et al. found that tumor stage is a significant prognostic factor for NPC [33]. Furthermore, clinical stage was previously found related to overall survival of patients [35]. A study of prostate cancer suggested higher $\mathrm{Cd}$ levels detected in advanced stages [49]. The advanced stage has a poor prognostic effect on local control rate and metastasis of the disease, whereas early stages have a better survival advantage [33,50].

Many studies have found Cd level as a risk factor for NPC [22,36,37], but few assessed the effect of $\mathrm{Cd}$ exposure on the survival and prognosis of NPC. One study investigated a head and neck cancer population in Tunisia, including 48 NPC patients and 97 laryngeal cancer patients; Cd level was higher in NPC patients than controls $(2.95 \pm 4.93$ vs. $0.74 \pm 1.15, p<0.001)$ [22]. Another study, also carried out in Tunisia, found higher $\mathrm{Cd}$ level in tumor tissues of patients with head and neck cancer (including 34 NPC patients, 45 laryngeal cancer patients) than in healthy tissues $(0.95 \pm 1.38$ vs. $0.22 \pm 0.43$, $p<0.001$ ) [36]. By adding a normal control population, it will also be significant to explore blood Cd level on the risk of NPC. Actually, we conducted a case-control study [37] previously to explore the association between Cd level and NPC risk, and found that Cadmium seemed to be a risk factor of NPC, and that high Cd exposure may promote the occurrence and development of NPC.These findings were relatively consistent. In the present study, we assessed the association between these factors and PFS with NPC for a better understanding of the prognostic process. $\mathrm{Cd}$, as a novel risk factor, should be specifically analyzed for its effect on NPC prognosis.

With the industrial production process, Cd (a common toxic metal pollutant) exists in water, soil, food and air [24]. Cd was first identified as a lung carcinogen in mechanism and epidemiological studies, and it exists widely in soft tissue [51]. Cd has certain toxic effects on breast, prostate, kidney and bone tissue by external ingestion and body accumulation [52]. Recent studies have also found high levels of blood Cd in patients with nasosinusal polyposis and NPC. In addition, in a case-control study, blood Cd level was higher in NPC cases than controls (OR $=3.42 ; 95 \%$ CI 1.86-6.30) [37]. Another study concluded high blood Cd level in cases [22], and the result was consistent with our previous research [37]. In this study, we found that high Cd level was related to $\mathrm{N}$ classification, clinical stage, 
smoking history and disease history in patients with NPC. High blood Cd level was significantly related to NPC progression, and Cd exposure may be associated with NPC prognosis.

Here, we found that high Cd level was related to short PFS. Moreover, multivariate analysis concluded $\mathrm{Cd}$ as an independent prognostic factor for NPC progression after adjustment for other factors, including sex, residence, family history of cancer, history of disease, alcohol drinking, smoking pack-years, clinical stage, and blood $\mathrm{Cd}$ level. Previous studies have found that $\mathrm{Cd}$ level isa risk factor for NPC development and is also associated with the prognosis of NPC [22].

There are many sources of metal exposure, and ingestion and inhalation are two major ways for metals to enter the body. Cd toxicity is well known and has a long half-life of approximately 20 years in an organism, so the concentration in body fluids must be monitored [53]. Kidney is the main accumulation organ of $\mathrm{Cd}$, but other soft tissues also show a certain accumulation effect, such as the prostate, lungs, and mucosa. Hence, the elevated incidence of respiratory and renal disease with $\mathrm{Cd}$ exposure is not surprising [54]. Cd has been classified as a carcinogen to humans by the International Agency for Research on Cancer for a long time, and relevant experiments have shown that subcutaneous injection of $\mathrm{Cd}$ into rodents causes local sarcomata formation [55-57]. One study found that battery workers had a high risk of NPC, caused by the long-term inhalation of large amounts of Cd particles that accumulate in the nose [54]. Anosmia is a kind of toxic disease caused by long-term exposure to high $\mathrm{Cd}$ levels [58]. Recent evidence showed that $\mathrm{Cd}$ entering the body mainly interfered with the gene synthesis and repair process, thus affecting the response to adverse effects and might contribute to chronic nasal inflammation [59]. Exposure to $\mathrm{Cd}$ in the external environment exceeds immunology function and results in tumor formation, and the process may explain the progression of NPC [60].

Because of $\mathrm{Cd}$ exposure and accumulation in the body, the respiratory function and metabolism of the body are changed. With exposure to different chemical poisons, the involved genes have different detoxification mechanisms, such as the enzyme cytochrome P450 (CYP2E1). The activity of CYP2E1 increased in mucosal cells induces the formation of free radicals and leads to cell injury. Additionally, it may interfere with gene regulation and cell differentiation. Because of different repair mechanisms of DNA, abnormal gene expression that encodes cell growth promotes the occurrence of tumors [22,61]. Normally, NPC occurs from the combined action of various mixtures, such as other metals or organic chemicals. Other studies have found that elevated blood levels of $\mathrm{Cd}$ result from an imbalance in $\mathrm{Zn} /$ magnesium ratio [62]. Several studies suggested a higher blood Cd level in NPC patients than healthy controls $(p<0.05)[22,37]$, so Cd could be a risk factor for NPC patients and may also affect the prognosis of patients and increase the chances of NPC progression.

Apart from occupational and environmental exposures, lifestyle habits such as smoking are a major factor in $\mathrm{Cd}$ intake. Many studies have shown that tobacco smoke exposure increases $\mathrm{Cd}$ level in the body $[63,64]$, and the Cd level is approximately 2 to 4 times higher in smokers than non-smokers [65]. We also found a strong positive correlation between $\mathrm{Cd}$ level and smoking history. Similarly, blood Cd level was significantly correlated with the number of daily cigarettes smoked and pack-years of smoking. Cd exposure is much higher from cigarette smoking than diet and air. In addition, we found that smoking pack-years isa risk factor of blood Cd level (OR $=4.83 ; 95 \% \mathrm{CI}$ 1.68-13.91; Table 3). Of note, the median blood Cd level was previously found much higher in smokers than non-smokers (1.02 vs. $0.51 \mathrm{ng} / \mathrm{mL}$ ) [63]. In our study, the blood Cd level was higher in smokers than non-smokers $(p=0.01)$. Smoking history was associated with short PFS on Kaplan-Meier but not multivariate analysis. Blood Cd level may mask the true effects of smoking. Further studies are needed to clarify whether smoking affects the prognosis of NPC.

We found that $\mathrm{Cd}$, as a novel risk factor, affected the prognosis of NPC, which indicates the danger of behaviors and environmental metal exposure to the human body to some extent. Intervening in $\mathrm{Cd}$ exposure may better guide patients to better prognosis and clinical practice. However, our study contains some limitations, such as the confounding effect of clinical stage on prognosis, and other factors that may have contributed to biased results. Firstly, this was not a case-control study, but a survival analysis of factors affecting the prognosis of NPC cases. We included NPC cases and observed the 
occurrence of endpoint events during follow-up, so the proportion of outcomes and non-outcomes could not be designed in advance. Due to the limited sample size, the results may be slightly biased. Further intensive studies based on well-designed methods and larger sample size are needed to examine the association. Secondly, although we did not collect accurate information about the dose of chemotherapy using cisplatin and paclitaxel on NPC patients, the effect of chemotherapy on the findings was less likely. Since all the patients we included received similar chemotherapy, this is most likely to have resulted in the attenuation of risk estimates toward the null between two groups. Hence, the results of the study were unaffected and reliable. Certainly, the effect of platinum $(\mathrm{Pt})$ used for chemotherapies on the prognosis of NPC patients also needsto be explored in future studies. Thirdly, to better reflect the effects of long-term and chronic exposure to metals, the sample tested should be urine; blood $\mathrm{Cd}$ is rapidly cleared by the kidneys, so it is often used as a biomarker of recent exposure [66]. However, high blood $\mathrm{Cd}$ level and long-term exposure are relatively consistent, so the level can also be used to reflect past exposure $[67,68]$. Therefore, the use of blood Cd level can reflect the human burden of $\mathrm{Cd}$.

\section{Conclusions}

In the present study, we assessed the effects of various factors on the prognosis and progression of NPC by measuring blood Cd level and clinicopathologic information. Cd level was an independent prognostic risk factor for NPC on multifactorial Cox regression analysis. Furthermore, high blood Cd level, male sex, smoking history and increasing pack-years as well as clinical stage were risk factors for short PFS with NPC. Subsequent studies are needed to clarify the effect of Cd exposure and other clinical factors on the prognosis and the progression of NPC.

Author Contributions: The co-authors contributed to the completion of this article together. Specifically, conceptualization, K.W. and T.D.; methodology, W.H.; software, S.Z.; validation, Y.H. and M.B.; formal analysis, T.D.; investigation, Y.H. and A.L.; resources, W.H. and M.H.; data curation, S.Z.; writing—original draft preparation, T.D.; writing-review and editing, T.D. and K.W.; supervision, T.D.; project administration, K.W. and T.D.; funding acquisition, K.W.

Funding: This work was supported by the Natural Science Foundation of Guangdong Province (No. 2016A030313071).

Acknowledgments: We thank anyone who contributed towards the article and provided professional writing services or materials. We also obtained permission to acknowledge all those mentioned. We thank Laura Smales (BioMedEditing, Toronto, Canada) for English language editing.

Conflicts of Interest: The authors declare no conflict of interest.

\section{References}

1. Ferlay, J.; Soerjomataram, I.; Dikshit, R.; Eser, S.; Mathers, C.; Rebelo, M.; Parkin, D.M.; Forman, D.; Bray, F. Cancer incidence and mortality worldwide: Sources, methods and major patterns in GLOBOCAN 2012. Int. J. Cancer 2015, 136, E359-E386. [CrossRef] [PubMed]

2. Jackson, C. Primary carcinoma of the nasopharynx. a table of cases. JAMA 1901, 37, 371-377. [CrossRef]

3. Digby, K.H. Nasopharyngeal carcinoma. Br. J. Surg. 1941, 28, 517-537. [CrossRef]

4. Ferlay, J.; Parkin, D.; Shin, H.; Forman, D.; Mathers, C.; Parkin, D.; Bray, F. Globocan 2000. Int. J. Cancer 2001, 94, 153-156.

5. Caponigro, F.F.; Longo, F.; Perri, F. Treatment approaches to nasopharyngeal carcinoma: A review. Anticancer Drugs 2010, 21, 471-477. [CrossRef] [PubMed]

6. Liu, A.W.; Huang, G.; Zeng, X.; Ma, X.; Zhou, Y.; Wang, C.; Cheng, A. Expression of the Annexin A1 gene is associated with suppression of growth, invasion and metastasis of nasopharyngeal carcinoma. Mol. Med. Rep. 2014, 10, 3059-3067. [CrossRef]

7. Henderson, B.E.; Louie, E.; Soohoo, J.J.; Buell, P.; Gardner, M.B. Risk factors associated with nasopharyngeal carcinoma. N. Engl. J. Med. 1976, 295, 1101-1106. [CrossRef]

8. Lee, A.W.; Ng, W.T.; Chan, Y.H.; Sze, H.; Chan, C.; Lam, T.H. The battle against nasopharyngeal cancer. Radiother. Oncol. 2012, 104, 272-278. [CrossRef] 
9. Nam, J.; Mclaughlin, J.K.; Blot, W.J. Cigarette Smoking, Alcohol, and Nasopharyngeal Carcinoma: A Case-Control Study Among U.S. Whites. J. Natl. Cancer Inst. 1992, 84, 619-622. [CrossRef]

10. Purdue, M.P.; Järvholm, B.; Bergdahl, I.A.; Hayes, R.B.; Baris, D. Occupational exposures and head and neck cancers among Swedish construction workers. Scand. J. Work Environ. Health 2006, 32, 270-275. [CrossRef]

11. Ulenbelt, P.; Lumens, M.E.; Géron, H.M.; Herber, R.F.; Broersen, S.; Zielhuis, R.L. Work hygienic behaviour as modifier of the lead air-lead blood relation. Int. Arch. Occup. Environ. Health 1990, 62, 203-207. [CrossRef] [PubMed]

12. Afridi, I.H.; Kazi, T.G.; Kazi, A.G.; Shah, F.; Wadhwa, S.K.; Kolachi, N.F.; Shah, A.Q.; Baig, J.A.; Kazi, N. Levels of arsenic, cadmium, lead, manganese and zinc in biological samples of paralysed steel mill workers with related to controls. Biol. Trace Elem. Res. 2011, 144, 164-182. [CrossRef] [PubMed]

13. Gil, F.; Hernández, A.F.; Márquez, C.; Femia, P.; Olmedo, P.; López-Guarnido, O.; Pla, A. Biomonitorization of cadmium, chromium, manganese, nickel and lead in whole blood, urine, axillary hair and saliva in an occupationally exposed population. Sci. Total Environ. 2011, 409, 1172-1180. [CrossRef] [PubMed]

14. Bernhard, D.; Rossmann, A.; Wick, G. Metals in cigarette smoke. Iubmb. Life 2005, 57, 805-809.

15. Eyre, H. Association. Preventing cancer, cardiovascular disease, and diabetes: A common agenda for the American Cancer Society, the American Diabetes Association, and the American Heart Association. Circulation 2004, 109, 3244-3255. [CrossRef] [PubMed]

16. Yeon, S.K.; Wook, K.J.; Sook, K.Y.; Eun, K.J.; Yeon, C.H.; Cha, L.K.Y. Changes in lipid peroxidation and antioxidant trace elements in serum of women with cervical intraepithelial neoplasia and invasive cancer. Nutr. Cancer 2003, 47, 126-130.

17. Kazi, G.T.; Memon, A.R.; Afridi, H.I.; Jamali, M.K.; Arain, M.B.; Jalbani, N.; Sarfraz, R.A. Determination of cadmium in whole blood and scalp hair samples of Pakistani male lung cancer patients by electrothermal atomic absorption spectrometer. Sci. Total Environ. 2008, 389, 270-276. [CrossRef] [PubMed]

18. Kazi, G.T.; Wadhwa, S.K.; Afridi, H.I.; Kazi, N.; Kandhro, G.A.; Baig, J.A.; Shah, A.Q.; Kolachi, N.F.; Arain, M.B. Interaction of cadmium and zinc in biological samples of smokers and chewing tobacco female mouth cancer patients. J. Hazard. Mater. 2010, 176, 985-991. [CrossRef] [PubMed]

19. Yuan, H.T.; Ie, B.L.; Tsai, K.Y.; Chang, T.K.; Chiang, C.T.; Su, C.C.; Hwang, Y.H. Possible association between nickel and chromium and oral cancer: A case-control study in central Taiwan. Sci. Total Environ. 2011, 409, 1046-1052. [CrossRef] [PubMed]

20. Wadhwa, K.S.; Kazi, T.G.; Kolachi, N.F.; Afridi, H.I.; Khan, S.; Chandio, A.A.; Shah, A.Q.; Kandhro, G.A.; Nasreen, S. Case-control study of male cancer patients exposed to arsenic-contaminated drinking water and tobacco smoke with relation to non-exposed cancer patients. Hum. Exp. Toxicol. 2011, 30, 2013-2022. [CrossRef]

21. Tandon, M.U.; Kapil, S.; Bahadur, S.; Dwivedi, S.N.; Pathak, P. Role of micro-nutrients and trace elements in carcinoma of larynx. J. Assoc. Physic. India 2000, 48, 995-998.

22. Khlifi, R.; Olmedo, P.; Gil, F.; Molka, F.T.; Hammami, B.; Ahmed, R.; Amel, H.C. Risk of laryngeal and nasopharyngeal cancer associated with arsenic and cadmium in the Tunisian population. Environ. Sci. Pollut. Res. 2014, 21, 2032-2042. [CrossRef] [PubMed]

23. Garnit, H.; Bouhlel, S.; Barca, D.; Chtara, C. Application of LA-ICP-MS to sedimentary phosphatic particles from Tunisian phosphorite deposits: Insights from trace elements and REE into paleo-depositional environments. Chem. Erd. 2012, 72, 127-139. [CrossRef]

24. Meeker, D.J.; Rossano, M.G.; Protas, B.; Diamond, M.P.; Puscheck, E.; Daly, D.; Paneth, N.; Wirth, J.J. Cadmium, Lead, and Other Metals in Relation to Semen Quality: Human Evidence for Molybdenum as a Male Reproductive Toxicant. Environ. Health Perspect. 2008, 116, 1473. [CrossRef] [PubMed]

25. Satarug, S.; Baker, J.R.; Urbenjapol, S.; Haswell-Elkins, M.; Reilly, P.E.; Williams, D.J.; Moore, M.R. A global perspective on cadmium pollution and toxicity in non-occupationally exposed population. Toxicol. Lett. 2003, 137, 65-83. [CrossRef]

26. Byrne, C.; Divekar, S.D.; Storchan, G.B.; Parodi, D.A.; Martin, M.B. Metals and breast cancer. J. Mammary Gland Biol. Neoplasia 2013, 18, 63-73. [CrossRef]

27. Akesson, A.; Julin, B.; Wolk, A. Long-term dietary cadmium intake and postmenopausal endometrial cancer incidence: A population-based prospective cohort study. Cancer Res. 2008, 68, 6435-6441. [CrossRef]

28. Kellen, E.; Zeegers, M.P.; Hond, E.D.; Buntinx, F. Blood cadmium may be associated with bladder carcinogenesis: The Belgian case-control study on bladder cancer. Cancer Detect. Prev. 2007, 31, 77-82. [CrossRef] 
29. Luckett, G.B.; Su, L.J.; Rood, J.C.; Fontham, E.T. Cadmium exposure and pancreatic cancer in south Louisiana. J. Environ. Public Health 2012, 2012, 180186. [CrossRef]

30. Julin, B.; Wolk, A.; Bergkvist, L.; Bottai, M.; Akesson, A. Dietary cadmium exposure and risk of postmenopausal breast cancer: A population-based prospective cohort study. Cancer Res. 2012, 72, 1459-1466. [CrossRef]

31. Garcia-Esquinas, E.; Pollan, M.; Tellez-Plaza, M.; Francesconi, K.A.; Goessler, W.; Guallar, E.; Umans, J.G.; Yeh, J.; Best, L.G.; Navas-Acien, A. Cadmium exposure and cancer mortality in a prospective cohort: The strong heart study. Environ. Health Perspect. 2014, 122, 363-370. [CrossRef] [PubMed]

32. Qu, Y.; Chen, Y.; Yu, H.; Zhao, Y.; Chen, G.; Bai, L.; Liu, D.; Su, H.; Wang, H. Survival and Prognostic Analysis of Primary Nasopharyngeal Carcinoma in North China. Clin. Lab. 2015, 61, 699. [CrossRef] [PubMed]

33. Cheng, H.S.; Yen, K.L.; Jian, J.J.; Tsai, S.Y.; Chu, N.M.; Leu, S.Y.; Chan, K.Y.; Tan, T.D.; Cheng, J.C.; Hsieh, C.Y. Examining prognostic factors and patterns of failure in nasopharyngeal carcinoma following concomitant radiotherapy and chemotherapy: Impact on future clinical trials. Int. J. Radiat. Oncol. Biol. Phys. 2001, 50, 717-726. [CrossRef]

34. Mao, P.Y.; Xie, F.Y.; Liu, L.Z.; Sun, Y.; Li, L.; Tang, L.L.; Liao, X.B.; Xu, H.Y.; Chen, L.; Lai, S.Z. Re-evaluation of 6th edition of AJCC staging system for nasopharyngeal carcinoma and proposed improvement based on magnetic resonance imaging. Int. J. Radiat. Oncol. Biol. Phys. 2009, 73, 1326-1334. [CrossRef] [PubMed]

35. Lai, Z.S.; Li, W.F.; Chen, L.; Luo, W.; Chen, Y.Y.; Liu, L.Z.; Sun, Y.; Lin, A.H.; Liu, M.Z.; Ma, J. How Does Intensity-Modulated Radiotherapy Versus Conventional Two-Dimensional Radiotherapy Influence the Treatment Results in Nasopharyngeal Carcinoma Patients? Int. J. Radiat. Oncol. Biol. Phys. 2011, 80, 661-668. [CrossRef] [PubMed]

36. Khlifi, R.; Olmedo, P.; Gil, F.; Hammami, B.; Chakroun, A.; Rebai, A.; Hamza-Chaffai, A. Arsenic, cadmium, chromium and nickel in cancerous and healthy tissues from patients with head and neck cancer. Sci. Total Environ. 2013, 452, 58-67. [CrossRef] [PubMed]

37. Peng, L.; Wang, X.; Huo, X.; Xu, X.; Lin, K.; Zhang, J.; Huang, Y.; Wu, K. Blood cadmium burden and the risk of nasopharyngeal carcinoma: A case-control study in Chinese Chaoshan population. Environ. Sci. Pollut. Res. Int. 2015, 22, 12323-12331. [CrossRef] [PubMed]

38. Zheng, L.; Wu, K.; Li, Y.; Qi, Z.; Han, D.; Zhang, B.; Gu, C.; Chen, G.; Liu, J.; Chen, S. Blood lead and cadmium levels and relevant factors among children from an e-waste recycling town in China. Environ. Res. 2008, 108, 15-20. [CrossRef]

39. Warren, W.G.; Reid, M.E.; Cummings, K.M.; Marshall, J.R. Author's reply to: Smoking at diagnosis and survival in cancer patients. Int. J. Cancer 2013, 132, 401-410. [CrossRef]

40. Xiao, W.W.; Huang, S.M.; Han, F.; Wu, S.X.; Lu, L.X.; Lin, C.G.; Deng, X.W.; Lu, T.X.; Cui, N.J.; Zhao, C. Local control, survival, and late toxicities of locally advanced nasopharyngeal carcinoma treated by simultaneous modulated accelerated radiotherapy combined with cisplatin concurrent chemotherapy: Long-term results of a phase 2 study. Cancer 2011, 117, 1874-1883. [CrossRef]

41. Lee, W.A.; Sze, W.M.; Au, J.S.; Leung, S.F.; Leung, T.W.; Chua, D.T.; Zee, B.C.; Law, S.C.; Teo, P.M.; Tung, S.Y. Treatment results for nasopharyngeal carcinoma in the modern era: The Hong Kong experience. Int. J. Radiat. Oncol. Biol. Phys. 2005, 61, 1107-1116. [CrossRef] [PubMed]

42. Salem, F.A.; Al-Zoubi, M.S.; Whitaker-Menezes, D.; Martinez-Outschoorn, U.E.; Lamb, R.; Hulit, J.; Howell, A.; Gandara, R.; Sartini, M.; Galbiati, F. Cigarette smoke metabolically promotes cancer, via autophagy and premature aging in the host stromal microenvironment. Cell Cycle 2013, 12, 818-825. [CrossRef] [PubMed]

43. Browman, P.G.; Wong, G.; Hodson, I.; Sathya, J.; Russell, R.; Mcalpine, L.; Skingley, P.; Levine, M.N. Influence of cigarette smoking on the efficacy of radiation therapy in head and neck cancer. N. Engl. J. Med. 1993, 328, 159. [CrossRef] [PubMed]

44. Browman, G.P.; Mohide, E.A.; Willan, A.; Hodson, I.; Wong, G.; Grimard, L.; MacKenzie, R.G.; El-Sayed, S.; Dunn, E.; Farell, S. Association between smoking during radiotherapy and prognosis in head and neck cancer: A follow-up study. Head Neck 2002, 24, 1031-1037. [CrossRef]

45. Chen, A.M.; Chen, L.M.; Vaughan, A.; Sreeraman, R.; Farwell, D.G.; Luu, Q.; Lau, D.H.; Stuart, K.; Purdy, J.A.; Vijayakumar, S. Tobacco smoking during radiation therapy for head-and-neck cancer is associated with unfavorable outcome. Int. J. Radiat. Oncol. Biol. Phys. 2011, 79, 414. [CrossRef] [PubMed]

46. Xiao, W.W.; Han, F.; Lu, T.X.; Chen, C.Y.; Huang, Y.; Zhao, C. Treatment outcomes after radiotherapy alone for patients with early-stage nasopharyngeal carcinoma. Int. J. Radiat. Oncol. Biol. Phys. 2009, 74, 1070. [CrossRef] 
47. Yeh, A.S.; Tang, Y.; Lui, C.C.; Huang, Y.J.; Huang, E.Y. Treatment outcomes and late complications of 849 patients with nasopharyngeal carcinoma treated with radiotherapy alone. Int. J. Radiat. Oncol. Biol. Phys. 2005, 62, 672-679. [CrossRef] [PubMed]

48. Chen, Y.C.; Han, F.; Zhao, C.; Lu, L.X.; Sun, Y.; Liu, X.F.; Lu, T.X. Treatment results and late complications of 556 patients with locally advanced nasopharyngeal carcinoma treated with radiotherapy alone. Br. J. Radiol. 2009, 82, 452-458. [CrossRef]

49. Chen, C.Y.; Pu, Y.S.; Wu, H.C.; Wu, T.T.; Lai, M.K.; Yang, C.Y.; Sung, F.C. Cadmium burden and the risk and phenotype of prostate cancer. BMC Cancer 2009, 9, 429. [CrossRef]

50. Liu, T.M.; Hsieh, C.Y.; Chang, T.H.; Lin, J.P.; Huang, C.C.; Wang, A.Y. Prognostic Factors Affecting the Outcome of Nasopharyngeal Carcinoma. Jpn. J. Clin. Oncol. 2003, 33, 501-508. [CrossRef]

51. Zuckerman, A.J. IARC Monographs on the Evaluation of Carcinogenic Risks to Humans. J. Clin. Pathol. 1995, 48, 691. [CrossRef]

52. Hartwig, A. Cadmium and Cancer; Springer: Dordrecht, The Netherlands, 2013; pp. 491-507.

53. Ferguson, J.E. Heavy Elements: Chemistry, e.i.a.h.e., The Heavy Elements: Chemistry, Environmental Impact, and Health Effects; Pergamon Press: Oxford, UK, 1990; p. 69.

54. Tord, K.; Lars, F.; Barbro, R. Mortality and cancer morbidity among cadmium-exposed workers. Environ. Health Perspect. 1979, 28, 199.

55. Robards, K.; Worsfold, P. Cadmium: Toxicology and analysis: A review. Analyst 1991, 116, 549-568. [CrossRef] [PubMed]

56. Seiler, H.G.; Sigel, H.; Sigel, A. Handbook of Toxicity of Inorganic Compounds; Taylor and Francis: Abingdon, UK, 1988; p. 1024.

57. Kazantzis, G. Induction of Sarcoma in the Rat by Cadmium Sulphide Pigment. Nature 1963, 198, 1213-1214. [CrossRef]

58. Friberg, L.; Piscator, M.; Nordberg, G.F.; Kjellstroem, T. Cadmium in the Environment, 2nd ed.; CRC Press: Boca Raton, FL, USA, 1974.

59. Collins, M.M.; Pang, Y.T.; Loughran, S.; Wilson, J.A. Environmental risk factors and gender in nasal polyposis. Clin. Otolaryngol. Allied Sci. 2002, 27, 314-317. [CrossRef] [PubMed]

60. Khlifi, R.; Olmedo, P.; Gil, F.; Chakroun, A.; Hammami, B.; Hamza-Chaffai, A. Heavy metals in normal mucosa and nasal polyp tissues from Tunisian patients. Environ. Sci. Pollut. Res. Int. 2015, 22, 463-471. [CrossRef]

61. Pearson, C.A.; Prozialeck, W.C. E-Cadherin, beta -Catenin and cadmium carcinogenesis. Med. Hypotheses 2001, 56, 573-581. [CrossRef]

62. Afridi, I.H.; Kazi, T.G.; Kazi, N.; Kandhro, G.A.; Baig, J.A.; Jamali, M.K.; Arain, M.B.; Shah, A.Q. Erratum: Interactions Between Cadmium and Zinc in the Biological Samples of Pakistani Smokers and Nonsmokers Cardiovascular Disease Patients. Biol. Trace Elem. Res. 2011, 139, 368. [CrossRef]

63. El, A.O.; Gokmen, I.G. Smoking habits and cadmium intake in Turkey. Biol. Trace Elem. Res. 2002, 88, 31.

64. Pappas, S.R.; Polzin, G.M.; Zhang, L.; Watson, C.H.; Paschal, D.C.; Ashley, D.L. Cadmium, lead, and thallium in mainstream tobacco smoke particulate. Food Chem. Toxicol. 2006, 44, 714-723. [CrossRef]

65. Galazyn-Sidorczuk, M.; Brzoska, M.M.; Moniuszko-Jakoniuk, J. Estimation of Polish cigarettes contamination with cadmium and lead, and exposure to these metals via smoking. Environ. Monit. Assess. 2008, 137, 481-493. [CrossRef] [PubMed]

66. Waalkes, P.M.; Coogan, T.P.; Barter, R.A. Toxicological principles of metal carcinogenesis with special emphasis on cadmium. Crit. Rev. Toxicol. 1992, 22, 175-201. [CrossRef] [PubMed]

67. Nriagu, J.O. Arsenic in the Environment, Part 2, Human Health and Ecosystem Effects; Advances in Environmental Science and Technology Series; Wiley: Hoboken, NJ, USA, 1994.

68. Järup, L.; Berglund, M.; Elinder, C.G.; Nordberg, G.; Vahter, M. Health effects of cadmium exposure-A review of the literature and a risk estimate. Scand. J. Work Environ. Health 1998, 24, 1-51. [PubMed]

(C) 2019 by the authors. Licensee MDPI, Basel, Switzerland. This article is an open access article distributed under the terms and conditions of the Creative Commons Attribution (CC BY) license (http://creativecommons.org/licenses/by/4.0/). 